Relations industrielles

Industrial Relations

\title{
Décisions rendues par le Conseil canadien des relations du travail
}

\section{Dianne Pothier}

Volume 39, numéro 4, 1984

URI : https://id.erudit.org/iderudit/050084ar

DOI : https://doi.org/10.7202/050084ar

Aller au sommaire du numéro

Éditeur(s)

Département des relations industrielles de l'Université Laval

ISSN

0034-379X (imprimé)

1703-8138 (numérique)

Découvrir la revue

Citer cet article

Pothier, D. (1984). Décisions rendues par le Conseil canadien des relations du travail. Relations industrielles / Industrial Relations, 39(4), 774-782.

https://doi.org/10.7202/050084ar
Résumé de l'article

Decisions rendues par le Conseil canadien des relations du travail
Tous droits réservés (C) Département des relations industrielles de l'Universite Laval, 1984
Ce document est protégé par la loi sur le droit d'auteur. L'utilisation des services d'Érudit (y compris la reproduction) est assujettie à sa politique d'utilisation que vous pouvez consulter en ligne.

https://apropos.erudit.org/fr/usagers/politique-dutilisation/ 


\title{
Décisions rendues par le Conseil canadien des relations du travail
}

\author{
Réorganisation structurelle d'entreprise
}

Le Conseil a refusé d'appliquer les dispositions de l'article 133 portant sur la question d'employeur unique du fait qu'une des entreprises en cause n'était plus de compétence fédérale, ce qui l'empêchait de rendre une ordonnance applicable dans l'immédiat ou à l'avenir.

Le Conseil a jugé qu'il y avait eu vente d'entreprise au sens de l'article $144 \mathrm{du}$ Code car la compagnie, afin de changer de convention collective, s'était soustraite d̀ la compétence fédérale en transférant les permis d'exploitation, le travail, les camions, les employés, la direction et l'achalandage à une de ses filiales à part entière. Aux termes de l'alinéa 144(2)c), la convention collective visant l'entreprise vendue continuait de s'appliquer non seulement après la vente, mais pendant celle-ci. Le Conseil a refusé d'appliquer les dispositions du paragraphe 144(3) portant sur la confusion lorsque le même syndicat était l'agent négociateur tant de l'employeur prédécesseur que de l'employeur successeur.

Section Locale 31 (General Drivers and Helpers) de la Fraternite des Teamsters, requerante, Commercial Truck Company Ltd. et Intermountain Transport Ltd., intimés, et Jack Barkwell et autres, employés intervenants.

Dossier du Conseil 560-77 et 585-98, décision du 1/ septembre 1984 (No. 480); Panel du Conseil: Hugh R. Jamieson, VicePrésident, MM. Lorne E. Shaffer et James D. Abson, Membres.

\section{FAITS SAILLANTS}

La Section locale 31 a présenté deux requêtes au Conseil. Elle a demandé en particulier que le Conseil fasse une déclaration d'employeur unique à l'égard de Commercial Truck Company Ltd. (Commercial) et de Intermountain Transport Ltd. (Intermountain), en vertu de l'article 133 du Code. Les employeurs ont soulevé une question d'ordre constitutionnel pour s'opposer à la requête, affirmant que Commercial ne relevait plus de la compétence fédérale ${ }^{1}$.

- Cette chronique a été rédigée par Dianne POTHIER, avocate, conseiller juridique auprès du Président du Conseil canadien des relations du travail.

Toute opinion pouvant découler de la présente chronique et exprimée par l'auteur en sus du texte officiel des décisions du C.C.R.T. ne lie pas ce dernier.

1 Conformément à la politique que le Conseil applique, lorsqu'une question constitutionnelle est soulevée, la procédure a été enregistrée lors de l'audience. 
Par ailleurs, la Section locale demandait que le Conseil déclare qu'il y avait eu vente d'entreprise au sens de l'article 144 du Code de Commercial à Intermountain. Le syndicat a prétendu que la convention collective de Commercial devrait s'appliquer à tous les employés de Intermountain.

Un groupe d'anciens employés de Commercial qui étaient devenus chauffeurs à contrat chez Intermountain était intervenu et s'était opposé aux requêtes du syndicat.

Intermountain était et est une filiale à part entière de Commercial. Les deux entreprises partageaient et partagent encore le même personnel de direction. Les requêtes portaient sur une série d'événements mettant en cause Commercial et Intermountain et qui étaient survenus entre février et juin 1984. Il n'a pas été contesté que, avant février 1984, tant Commercial que Intermountain relevaient de la compétence fédérale puisqu'elles exerçaient des activités extra-provinciales dans le secteur du camionnage. Les principales activités extra-provinciales de Commercial s'exerçaient entre la Colombie-Britannique et les Etats-Unis, notamment entre Vancouver et Seattle. Intermountain exerçait également ses activités entre ces deux villes, mais seulement en cas de surplus de travail, et pour ce faire, elle utilisait les permis d'exploitation de Commercial. Avant 1984, Intermountain exerçait principalement ses activités extra-provinciales entre la Colombie-Britannique et l'Alberta.

La Section locale 31 a été accréditée à l'égard des employés de Commercial en 1971. Elle avait été volontairement reconnue par Intermountain au moment où Commercial avait fait l'acquisition de cette dernière entreprise, vers la fin des années $70^{2}$. Commercial était membre d'une association d'employeurs appelée la Transport Labour Relations (TLR), mais ce n'était pas le cas de Intermountain. Commercial était par conséquent liée par la convention cadre sur le transport, soit la Master Freight and Cartage Agreement, qui visait aussi ses concurrents effectuant le même trajet. La convention collective de Commercial était conçue en fonction d'entreprises dont les employés conduisaient des camions appartenant à la compagnie. La convention collective de Intermountain, en revanche, était conçue à l'intention des propriétaires-exploitants.

Commercial ainsi que ses concurrents avaient éprouvé des difficultés financières dans le transport de marchandises entre Vancouver et Seattle. Les membres de TLR désiraient obtenir des concessions de la Section locale 31. Cette dernière avait fait des arrangements avec deux concurrents de Commercial: elle avait conclu une entente concernant l'achat d'actions avec l'un et approuvé un tarif de $\$ 150$ avec l'autre pour chaque trajet. Commercial n'avait pas conclu d'entente avec la Section locale 31. Au lieu de cela, elle avait court-circuité la Section locale 31 et avait négocié directement avec les chauffeurs.

2 Deux autres sections locales des Teamsters, la Section locale 362 (établie en Alberta) et la Section locale 395 (établie en Saskatchewan), ont également été reconnues volontairement par Intermountain. Cependant, en 1984, Intermountain exerçait ses activités exclusivement à partir de la Colombie-Britannique, de sorte que la Section locale 31, établie en ColombieBritannique, était la seule section locale d̀ laquelle elle avait réellement affaire. 
Au début de 1984, les chauffeurs de Commercial avaient le choix entre être mis à pied pour une période indéterminée par Commercial ou devenir chauffeurs à contrat chez Intermountain. La plupart des chauffeurs avaient choisi la seconde solution. Les autres avaient été mis à pied par Commercial. Le syndicat avait déposé des griefs concernant ces mises à pied.

Dès février 1984, Commercial avait vendu ses camions à une filiale et les avait loués à plusieurs de ses anciens chauffeurs qui étaient devenus chauffeurs à contrat chez Intermountain. Le processus de transfert avait pris fin en avril 1984. Les nouveaux chauffeurs avaient été intégrés aux propriétaires-exploitants originaires de Intermountain. La convention collective de Intermountain était appliquée d tous les employés. Intermountain avait pris en charge les activités extra-provinciales exécutées auparavant par Commercial et avait continué d'utiliser les factures communes. En avril 1984, Commercial n'effectuait plus à son propre compte de trajets à l'extérieur de la province, même si ses permis d'exploitation lui permettaient encore de le faire. Commercial avait demandé que ses permis d'exploitation extraprovinciaux soient transférés à Intermountain. Les autorités américaines ainsi que celles de la Colombie-Britannique avaient toutes deux effectué ce transfert en juin 1984. Au cours de l'audience, le Conseil a indiqué qu'en raison des éléments de preuve présentées par le syndicat même, il était convaincu que Commercial ne relevait plus de la compétence fédérale.

Le Conseil a conclu que l'employeur avait pris toutes ces mesures en ce qui a trait au trajet Vancouver-Seattle, dans le dessein de se dérober à la convention collective de Commercial et de n'être plus assujetti qu'à celle de Intermountain.

\section{QUESTIONS SOULEVÉES}

Le Conseil a été appelé à juger s'il pouvait ou devait faire une déclaration d'employeur unique en vertu de l'article 133, à effet rétroactif seulement, dans le cas où des facteurs constitutionnels l'empêchaient de faire une déclaration applicable dans l'immédiat ou à l'avenir.

Par ailleurs, les circonstances de cette affaire permettent-elles d'invoquer les dispositions de l'article 144 portant sur la vente d'une entreprise, et dans l'affirmative, quelles seraient les conséquences?

Le Conseil a, de sa propre initiative, soulevé la question de savoir si les tentatives faites par un employeur en vue de changer de convention collective en ne tenant pas compte de l'une des conventions collectives en vigueur, en court-circuitant le syndicat et en passant des contrats individuels avec les employés équivalaient à s'ingérer dans la présentation d'un syndicat, ce qui constitue une infraction à l'alinéa 184(1)a).

\section{PRINCIPAUX MOTIFS DE DÉCISION}

\section{L'article 133}

L'article 133 se lit comme suit: 
133. Lorsque le Conseil est d'avis que des entreprises fédérales associees ou connexes sont exploitées par deux employeurs ou plus qui assument en commun le contrôle ou la direction, il peut, après avoir donné aux employeurs la possibilité raisonnable de présenter des observations, déclarer, par ordonnance, qu'à toutes fins de la présente Partie ces employeurs ainsi que les entreprises exploitées par eux que l'ordonnance spécifie, constituent respectivement un employeur unique et une entreprise fédérale unique.

Bien que le syndicat ait donné la priorité à sa requête en vertu de l'article 133, le Conseil ne s'est pas étendu sur cet aspect de l'affaire. Sa conclusion selon laquelle Commercial ne relevait plus de la compétence fédérale faisait qu'il n'était plus compétent du point de vue constitutionnel pour accepter de faire, en vertu de l'article 133, une déclaration visant Commercial et applicable dans l'immédiat ou à l'avenir. Le syndicat a néanmoins demandé une déclaration à effet rétroactif afin de donner suite à des griefs à l'encontre de Intermountain à la suite de prétendues infractions à la convention collective de Commercial. L'avocat de Commercial a affirmé que la loi ne conférait pas au Conseil le pouvoir de faire une déclaration à effet rétroactif à moins que cette déclaration ne s'applique également dans l'immédiat ${ }^{3}$.

Le Conseil n'a pas jugé nécessaire de déterminer s'il avait le pouvoir, de par la loi, de faire une déclaration au seul effet rétroactif en vertu de l'article 133, parce que, en vertu de son pouvoir discrétionnaire, il avait décidé qu'une déclaration de ce genre ne s'imposait pas.

...The union is seeking a declaration covering what is, essentially, a transition period. Commercial is now out of the picture as far as this Board is concerned. Section 133 is designed to deal with on-going situations, not situations where one employer moves out and the other moves in. Such circumstances are more appropriately considered under the sale of business provisions of s. $144 . \ldots$

\section{L'article 144}

Le paragraphe 144(1) se lit comme suit:

144.(1) Au présent article,

«entreprise» désigne une entreprise fédérale et s'entend d'une partie d'une telle entreprise;

«vente», relativement dे une entreprise, comprend la location, le transfert et tout autre acte d'aliénation de l'entreprise.

Les considérations d'ordre constitutionnel découlant de l'article 133 ne s'appliquaient pas à l'article 144. Cette disposition n'a de conséquences que pour l'employeur successeur et il était incontestable que Intermountain, le prétendu employeur successeur, relevait de la compétence fédérale.

3 Il a été déjà établi que le Conseil pouvait faire une déclaration à effet rétroactif en vertu de l'article 133, si cette déclaration s'applique également au moment où elle est faite (North Canada Air Ltd., [1981] 2 C.F. 399 (C.A.). 
Le Conseil n'a pas eu de difficulté à conclure qu'il y avait eu vente d'entreprise, soit celle de Commercial à Intermountain, au sens de l'article 144. Il existait un lien évident entre Commercial et Intermountain ${ }^{4}$, comme en témoignait en particulier le transfert des permis d'exploitation et des camions, ce qui revient à dire qu'il y a eu transfert des biens essentiels. Le Conseil a accordé une importance particulière au transfert des permis d'exploitation.

... The strongest single indicator of the sale of a business in the present case is the transfer of running rights. In the trucking industry running rights are the key element of a company's business. Without running rights, the company has nothing. Although Intermountain had previously been able to use Commercial's running rights, it had to share them as the junior partner. Now it has them all to itself. That is certainly a significant acquisition.

Pour ce qui est des camions, bien que Intermountain n'en soit pas elle-même propriétaire, elle jouit de leur usage exclusif et c'est la seule chose qui importe réellement.

Il y a eu bien plus qu'un transfert des biens. Il y a eu transfert des compétences au niveau de la direction. En effet, la direction commune de Commercial et de Intermountain consacre maintenant davantage de temps à cette dernière qu'auparavant. Au-dessous du niveau de la direction, il y a eu transfert en bloc des employés de Commercial à Intermountain. Ce dernier transfert n'est pas le fait d'une coïncidence, mais découle d'un effort volontaire en vue de garder le même effectifs'. De plus, Intermountain a continué de compter sur l'achalandage de Commercial en utilisant les factures communes, par exemple. Un des facteurs les plus importants, du point de vue des faits, est qu'il existe une continuité dans le travail exécuté. Le Conseil a fait remarquer que les clients n'auraient pas remarqué vraiment le changement.

Comme la portion extra-provinciale des activités de Commercial constituait auparavant une entité, elle était cohérente et séparable au point de constituer une «partie» d'une entreprise au sens de l'article $144^{6}$.

Le Conseil a conclu son étude sur la question de savoir s'il y avait eu ou non vente d'entreprise par les remarques suivantes:

Counsel for the employers submits that there has been no sale of a business because Intermountain was already in the business. Intermountain simply expanded its business. It is certainly accurate to say that Intermountain has expanded its business, but the point is it has done so through an acquisition from

4 Ce quorum du Conseil avait confirmé la nécessité d'un lien dans l'affaire Freight Emergency Services, (1984) 84 CLLC \16,031. Dans cette affaire, le quorum avait effectué une longue étude de la jurisprudence portant sur les ventes d'entreprises, étude qu'il n'a pas été jugé nécessaire de reprendre ici.

5. Le Conseil a fait remarquer que même l'absence d'un transfert d'employés ne signifie pas nécessairement qu'il n'y a pas eu vente (voir l'affaire Radio CJYQ Limited (1978), 26 di 576; et [1978] 1 Can LRBR 565), en général l'existence d'un transfert permet de conclure qu'il y a eu vente.

6 Reimer Express Lines Limited et Bernshine Mobile Maintenance Ltd. (1984), 84 CLLC \$ 16,036 . 
Commercial. The running rights, the work, the trucks, the employees, the management, and the goodwill have all been transferred to Intermountain by Commercial. They have been transferred not piece by piece, but as a whole, as $a$ «going concern»". Although any one of these in isolation would not likely constitute a sale of business, the totality of what has been transferred from Commercial leads inescapably to the conclusion of a sale of business under s. 144.

Quelles sont les conséquences d'une vente? L'alinéa 144(2)c) peut nous éclairer sur cette question.

144.(2) Sous réserve du paragraphe (3), lorsqu'un employeur vend son entreprise,

a) un syndicat qui est l'agent négociateur des employés travaillant dans l'entreprise demeure leur agent négociateur;

b) un syndicat qui a présenté une demande d'accréditation visant des employés travaillant dans l'entreprise avant la date de la vente peut, sous réserve de la présente Partie, être accrédité par le Conseil à titre d'agent négociateur de ces employés;

c) toute convention collective qui, à la date de la vente, est applicable aux employés travaillant dans l'entreprise lie la personne à laquelle celle-ci est vendue; et

d) la personne d̀ laquelle l'entreprise est vendue devient partie à toute procédure en instance d̀ la date de la vente, qui a été engagée en vertu de la présente Partie et qui concerne les employés travaillant dans l'entreprise ou leur agent négociateur.

L'expression «dans l'entreprise», dans l'alinéa 144(2)c), se rapporte manifestement à l'entreprise vendue. Par conséquent, la convention de Commercial continue de s'appliquer à Intermountain, du moins à la partie qu'elle a acquise de Commercial.

La question du moment à partir duquel la convention de Commercial doit s'appliquer à Intermountain revêt également une certaine importance. L'alinéa 144(2)c) indique clairement que cette disposition vise à protéger les droits conférés par la convention collective, outre les droits d'accréditation mêmes. Elle est destinée à contrecarrer les tentatives faites par les employeurs en vue de miner les droits de négociation, ce que Commercial essayait précisément de faire.

Especially when s. 144(2)c) is read in the context of the rest of s. 144(2), it is clear that the collective agreement is meant to flow through without hiatus. It is not only after the sale that the collective agreement is binding on the successor, but also during the very process of the sale. Otherwise, the collective agreement could be completely undermined and the protection afforded by

7 La notion d'entreprise en tant qu'affaire «en activité» a été adoptée par le Conseil réuni en séance plénière, dans Terminus Maritime Inc. (1983), 50 di 178. 
s. $144(2)$ c) rendered illusory. The who and how of the selection of employees in the successor business is the most obvious example of where it is important that the collective agreement apply to the process of the sale. ... ${ }^{8}$

Par conséquent, Intermountain était liée par la convention collective de Commercial pendant tout le temps que la vente avait lieu, même s'il n'incombait pas au Conseil de déterminer si elle s'y était conformée.

Il a été plus difficile d'appliquer le paragraphe 144(3).

144(3) Lorsqu'un employeur vend son entreprise et que le groupe de ses employés et celui des employés de l'employeur auquel l'entreprise a été vendue sont confondus,

a) le Conseil peut, à la demande de tout syndicat concerné,

(i) décider si les employés concernés constituent une ou plusieurs unités habiles à négocier collectivement,

(ii) déterminer quel syndicat sera l'agent négociateur des employés de chacune de ces unités, et

(iii) modifier, dans la mesure où il l'estime nécessaire, tout certificat délivré $\grave{a}$ un syndicat ou la description d'une unité de négociation figurant dans une convention collective;

b) une convention collective qui concerne les employés d'une unité jugée, en application de l'alinéa a), habile à négocier collectivement et qui lie le syndicat qui, par décision du Conseil est l'agent négociateur de cette unité de négociation, continue de lier ce syndicat; et

c) l'une des parties à une convention collective mentionnée à l'alinéa b) peut, da tout moment, passé un délai de soixante jours à partir de la date à laquelle le Conseil a statué sur une demande à lui présentée en vertu de l'alinéa a), demander à celui-ci de rendre une ordonnance l'autorisant à signifier à l'autre partie une mise en demeure de négocier collectivement.

Le syndicat a effectivement présenté une requête en vertu de l'alinéa 144(3)a), et toutes les parties ont convenu qu'il y avait eu confusion.

D'ordinaire, dans le cas de confusion, la question en jeu est celle de savoir quel syndicat représentera les employés. Il s'agit de l'objet du paragraphe 144(3). La question de la convention collective applicable découle de la réponse à la première question. Or, la présente affaire a une caractéristique unique car le même syndicat était agent négociateur tant de l'employeur prédécesseur que de l'employeur successeur. Il suffit donc de déterminer quelle convention collective doit s'appliquer, mais dans les circonstances le paragraphe 144(3) ne renferme aucune indication sur les critères qui doivent présider à ce choix.

8 La Commission des relations de travail de l'Ontario a adopté une position semblable dans l'affaire Emrick Plastics Inc., [1982] 3 Can LRBR 163. 
En outre, le Conseil a jugé que le fait de choisir une convention plutôt que l'autre présenterait des difficultés. Le fait de dire que seule la convention de Intermountain pouvait s'appliquer équivaudrait à sanctionner les tentatives faites en vue de contourner la convention de Commercial. Par contre, prétendre que seule la convention de Commercial doit rester en vigueur aurait pour conséquence d'attribuer à la Section locale 31 une convention qu'elle n'avait pas pu négocier en ce qui concerne les employés originaux de Intermountain. La question du type de convention approprié dans le cas de Intermountain devait être résolue par des négociations entre la Section locale 31 et cette entreprise, et le Conseil ne désirait pas faire pencher la balance en faveur de l'une ou de l'autre.

Le Conseil a conclu que l'affaire devait être tranchée conformément à l'alinéa 144(2)c), c'est-à-dire que la convention de Commercial devait s'appliquer à la partie de Intermountain acquise de Commercial. Pour ce qui est du reste de l'entreprise, la convention de Intermountain devait s'appliquer:

Since s. 144(3) is not really designed to cover the circumstances of this case, we do not think it advisable to try to stretch it to make it fit. The application of s. 144(3)a) is a matter of Board discretion ('the Board may'). We accordingly exercise our discretion not to apply s. 144(3)a), with the result that the rest of s. 144(3) and (4) are not invoked.

\section{L'alinéa 184(1)a)}

L'alinéa 184(1)a) se lit comme suit:

184(1) Nul employeur et nulle personne agissant pour le compte d'un employeur ne doit

a) participer à la formation ou d̀ l'administration d'un syndicat ou à la représentation des employés par un syndicat, ni s'y ingérer;

Bien que le syndicat n'ait déposé aucune plainte alléguant une violation de l'alinéa 184(1)a), le Conseil a formulé quelques brefs commentaires sur ce sujet. Il a toutefois indiqué que ses constatations ne constituaient pas une conclusion définitive puisque ni les preuves, ni les argumentations n'étaient reliées a l'alinéa 184(1)a), mais il croyait que les éléments de preuve dont il était saisi démontraient que cette disposition avait été violée ${ }^{9}$.

The negotiation of collective agreement is at the core of a union's representation of employees. For an employer to ignore a collective agreeement, by-pass the union, and make individual contracts with employees is direct interference

9 Le Conseil a établi une distinction entre l'affaire Reimer, supra, dans laquelle il avait conclu qu'il n'y avait pas eu violation de l'alinéa 184(1)a), même si l'employeur avait cédé du travail à l'extérieur pour éviter d'avoir à se conformer à une convention collective qu'il jugeait trop onéreuse, car dans cette affaire, la relation d'emploi avait été sevrée, ce qui n'est pas le cas de la présente affaire. Voir également Kennedy Lodge Inc. et al., décision non encore rapportée, dossiers 1909-83-U et 1910-83-R, 18 juillet 1984 (Ontario-C.R.T.O.). 
with a union's representation rights (see: Manitoba Pool Elevators (1980), 42 di 27; [1981] I Can LRBR 44, s. 28 application dismissed [1981] CLLC 14,160 (FCA); 39 N.R. 387). This is so even if the employees are subsequently covered under another collective agreement with the same union. An employer cannot pick and choose whatever collective agreement it likes. Manipulation aimed at avoiding collective agreements altogether would obviously be a more serious violation, but we conclude there can be a violation when there is a shift from one collective agreement to another. If an employer finds a collective agreement no longer economically viable, it is to the union that the employer must look for relief.

Since no complaint has been made by Local 31 (and since it is now too late to make one) we do not wish to belabour the point. We mention it only to explain the constraints under which the Board is placed. If there had been a complaint, the Board could have considered whether it was appropriate, as a remedy, to order a rollback of the leasing of the trucks. Without a complaint, though, the Board is powerless to do anything of this nature.

\section{DECISION}

Le Conseil a rejeté la requête présentée en vertu de l'article 133, mais a accepté de faire une déclaration selon laquelle il y avait eu vente d'entreprise, soit celle de Commercial à Intermountain, en vertu de l'article 144. La requête présentée par le syndicat en vertu de l'alinéa 144(3)a) a cependant été rejetée.

\section{NEW ZEALAND JOURNAL OF INDUSTRIAL RELATIONS}

\section{EDITOR: Peter Brosnan}

Industrial Relations Centre, Victoria University of Wellington, New Zealand

\begin{tabular}{lll}
\hline Vol. $9 \mathrm{~N}^{\circ} 2$ & Contents & August 1984 \\
\hline
\end{tabular}

\section{ARTICLES}

Managerial unionism and the law in New Zealand

Keith D. Binnie and David F. Smith

In recognition of skill: the growth of qualification payments, $1960-1980$

Full circle: Australian wage determination 1982-1984

\section{COMMENTARY}

Campaign for the return to free wage bargaining REPORT

The 1984 Federation of Labour conference. REVIEWS CHRONICLE APOLOGY
Pat Walsh

David H. Plowman

Ken Douglas

Raymond Harbridge

Published three times a year (April, August and December) by the New Zealand Institute of Industrial Relations Research Inc.

Subscriptions should be sent to Fay Thomson, Business Manager, Box 28093, Kelburn, Wellington.

Editorial correspondence: Should be addressed to David Smith, Editor, Department of Business Administration, Victoria University of Wellington, Private Bag, Wellington New Zealand. 